\title{
Reproducing Kernels for Hardy and Bergman Spaces of the Upper Half Plane
}

Job O. Bonyo ${ }^{1 *}$

\section{Abstract}

Using invertible isometries between Hardy and Bergman spaces of the unit disk $\mathbb{D}$ and the corresponding spaces of the upper half plane $\mathbb{U}$, we determine explicitly the reproducing kernels for the Hardy and Bergman spaces of $\mathbb{U}$. As a consequence, we obtain the duality relations for the reflexive Hardy and Bergman spaces of the half plane $\mathbb{U}$.

Keywords: Bergman projection, Duality, Hardy and Bergman spaces, Reproducing kernel, Szegö projection. 2010 AMS: Primary 30H10, 30H20, 47B33

${ }^{1}$ Department of Pure and Applied Mathematics, Maseno University, P.O. Box 333-40105, Maseno, Kenya, ORCID: 0000-0002-6442-4211 *Corresponding author: jobbonyo@maseno.ac.ke

Received: 8 October 2019, Accepted: 13 February 2020, Available online: 25 March 2020

\section{Introduction and Preliminary results}

Let $\mathbb{C}$ be the complex plane. The set $\mathbb{D}=\{z \in \mathbb{C}:|z|<1\}$, is called the (open) unit disc. Let $d A$ denote the area measure on $\mathbb{D}$, and for $\alpha \in \mathbb{R}, \alpha>-1$, we define a positive Borel measure $d m_{\alpha}$ on $\mathbb{D}$ by $d m_{\alpha}(z)=\left(1-|z|^{2}\right)^{\alpha} d A(z)$. On the other hand, the set $\mathbb{U}=\{\omega \in \mathbb{C}: \mathfrak{I}(\omega)>0\}$ denotes the upper half of the complex plane $\mathbb{C}$, and where $\mathfrak{I}(\omega)$ stands for the imaginary part of $\omega$. Also, $\mathfrak{R}(\omega)$ shall denote the real part of the complex number $\omega$. For $\alpha>-1$, we define a weighted measure on $\mathbb{U}$ by $d \mu_{\alpha}(\omega)=(\mathfrak{I}(\omega))^{\alpha} d A(\omega)$. The Cayley transform $\psi(z):=\frac{i(1+z)}{1-z}$ maps the unit disc $\mathbb{D}$ conformally onto the upper half-plane $\mathbb{U}$ with inverse $\psi^{-1}(\omega)=\frac{\omega-i}{\omega+i}$ mapping $\mathbb{U}$ conformally onto $\mathbb{D}$.

For an open subset $\Omega$ of $\mathbb{C}$, let $\mathscr{H}(\Omega)$ denote the Fréchet space of analytic functions $f: \Omega \rightarrow \mathbb{C}$ endowed with the topology of uniform convergence on compact subsets of $\Omega$. Let $\operatorname{Aut}(\Omega) \subset \mathscr{H}(\Omega)$ denote the group of biholomorphic maps $f: \Omega \rightarrow \Omega$. For $1 \leq p<\infty$, the Hardy spaces of the upper half plane, $H^{p}(\mathbb{U})$, are defined as

$$
H^{p}(\mathbb{U}):=\left\{f \in \mathscr{H}(\mathbb{U}):\|f\|_{H^{p}(\mathbb{U})}:=\sup _{y>0}\left(\int_{-\infty}^{\infty}|f(x+i y)|^{p} d x\right)^{1 / p}<\infty\right\},
$$

while the Hardy spaces of the unit disc, $H^{p}(\mathbb{D})$, by

$$
H^{p}(\mathbb{D}):=\left\{f \in \mathscr{H}(\mathbb{D}):\|f\|_{H^{p}(\mathbb{D})}^{p}:=\sup _{0<r<1} \frac{1}{2 \pi} \int_{-\pi}^{\pi}\left|f\left(r e^{i \theta}\right)\right|^{p} d \theta<\infty\right\} .
$$

We note that every function $f \in H^{p}(\mathbb{U})$ (or $H^{p}(\mathbb{D})$ ) has non-tangential boundary values almost everywhere on $\partial \mathbb{U}($ or $\partial \mathbb{D}$ ). In particular, $H^{p}$-functions my be identified with their boundary values and with this convention,

$$
\|f\|_{H^{p}(\mathbb{U})}=\left(\int_{-\infty}^{\infty}|f(x)|^{p} d x\right)^{\frac{1}{p}}
$$


and respectively,

$$
\|f\|_{H^{p}(\mathbb{D})}=\left(\int_{0}^{2 \pi}\left|f\left(e^{i \theta}\right)\right|^{p} d \theta\right)^{\frac{1}{p}} .
$$

On the other hand, for $1 \leq p<\infty, \alpha>-1$, the weighted Bergman spaces of the upper half plane, $L_{a}^{p}\left(\mathbb{U}, \mu_{\alpha}\right)$, are defined by

$$
L_{a}^{p}\left(\mathbb{U}, \mu_{\alpha}\right):=\left\{f \in \mathscr{H}(\mathbb{U}):\|f\|_{L_{a}^{p}\left(\mathbb{U}, \mu_{\alpha}\right)}=\left(\int_{\mathbb{U}}|f(z)|^{p} d \mu_{\alpha}(z)\right)^{\frac{1}{p}}<\infty\right\},
$$

while the corresponding spaces of the disc, $L_{a}^{p}\left(\mathbb{D}, m_{\alpha}\right)$, by

$$
L_{a}^{p}\left(\mathbb{D}, m_{\alpha}\right):=\left\{f \in \mathscr{H}(\mathbb{D}):\|f\|_{L_{a}^{p}\left(\mathbb{D}, m_{\alpha}\right)}=\left(\int_{\mathbb{D}}|f(z)|^{p} d m_{\alpha}(z)\right)^{\frac{1}{p}}<\infty\right\} .
$$

In particular, $L_{a}^{p}\left(\mathbb{U}, \mu_{\alpha}\right)=L^{p}\left(\mathbb{U}, \mu_{\alpha}\right) \cap \mathscr{H}(\mathbb{U})$ and $L_{a}^{p}\left(\mathbb{D}, m_{\alpha}\right)=L^{p}\left(\mathbb{D}, m_{\alpha}\right) \cap \mathscr{H}(\mathbb{D})$, where $L^{p}\left(\mathbb{U}, \mu_{\alpha}\right)$ or simply $L^{p}\left(\mu_{\alpha}\right)$ $\left(L^{p}\left(\mathbb{D}, m_{\alpha}\right)\right.$ or simply $\left.L^{p}\left(m_{\alpha}\right)\right)$ denotes the classical Lebesgue spaces associated with the weighted measure $\mu_{\alpha}$, and respectively $m_{\alpha}$. It is important to note that the case $\alpha=0$ yields the (unweighted) Bergman spaces.

As noted in [1] in the case of the disc, the Hardy space $H^{p}(\mathbb{U})$ behaves in many ways as the limiting case of $L_{a}^{p}\left(\mathbb{U}, \mu_{\alpha}\right)$ as $\alpha \rightarrow-1^{+}$. Therefore, we shall let $X$ denote either the Hardy space $H^{p}(\mathbb{U})$ or the weighted Bergman space $L_{a}^{p}\left(\mathbb{U}, \mu_{\alpha}\right)$, and we associate with each $X$, a parameter $\gamma=\frac{\alpha+2}{p}$, where $\alpha=-1$ in the case that $X=H^{p}(\mathbb{U})$. Also, we shall let $X(\mathbb{D})$ denote the corresponding spaces of analytic functions of the unit disc $\mathbb{D}$. Therefore, we formulate the growth conditions for Hardy and Bergman spaces simultaneously in the next results; while known, we provide much simpler proofs. But first we give the following result which gives the isometries between the spaces $X$ and $X(\mathbb{D})$.

Proposition 1.1. Let $f \in X$, and define $S_{\psi} f=\left(\psi^{\prime}\right)^{\gamma} f \circ \psi$. Then $S_{\psi}: X \rightarrow X(\mathbb{D})$ is continuous with inverse $S_{\psi^{-1}} g=$ $\left(\left(\psi^{-1}\right)^{\prime}\right)^{\gamma} g \circ \psi^{-1}$. In fact, if $X=H^{p}(\mathbb{U})$, then $S_{\psi}$ is an isometry, and, in the case $X=L_{a}^{p}\left(\mathbb{U}, \mu_{\alpha}\right),\left\|S_{\psi} f\right\|_{L_{a}^{p}\left(\mathbb{D}, m_{\alpha}\right)}=$ $2^{\alpha / p}\|f\|_{L_{a}^{p}\left(\mathbb{U}, \mu_{\alpha}\right)}$.

Moreover, $S_{\psi^{-1}}$ is an isometry on $H^{p}(\mathbb{D})$, and if $X(\mathbb{D})=L_{a}^{p}\left(\mathbb{D}, m_{\alpha}\right)$, then

$\left\|S_{\psi^{-1}} g\right\|_{L_{a}^{p}\left(\mathbb{U}, \mu_{\alpha}\right)}=2^{-\alpha / p}\|g\|_{L_{a}^{p}\left(\mathbb{D}, m_{\alpha}\right)}$.

In particular, $S_{\psi}^{-1}=S_{\psi^{-1}}$ in the setting of Bergman spaces as well as Hardy spaces.

Proof. First, we suppose that $X=L_{a}^{p}\left(\mathbb{U}, \mu_{\alpha}\right)$. Let $f \in L_{a}^{p}\left(\mathbb{U}, \mu_{\alpha}\right)$, then change of variables yields

$$
\begin{aligned}
\|f\|_{L_{a}^{p}\left(\mu_{\alpha}\right)} & =\int_{\mathbb{U}}|f(\omega)|^{p}(\mathfrak{I}(\omega))^{\alpha} d A(\omega) \\
& =\int_{\mathbb{D}}|f(\psi(z))|^{p}(\mathfrak{I}(\psi(z)))^{\alpha}\left|\psi^{\prime}(z)\right|^{2} d A(z),
\end{aligned}
$$

and $\mathfrak{I}(\psi(z))=\frac{\left(1-|z|^{2}\right)}{2}\left|\psi^{\prime}(z)\right|$. Thus $\|f\|_{L_{a}^{p}\left(\mu_{\alpha}\right)}^{p}=2^{-\alpha}\left\|S_{\psi} f\right\|_{L_{a}^{p}\left(m_{\alpha}\right)}^{p}$.

For the case $X=H^{p}(\mathbb{U})$, we may identify $f \in X$ with its boundary values. Then change of variables yields

$$
\begin{aligned}
\|f\|_{H^{p}(\mathbb{U})}^{p} & =\int_{\mathbb{R}}|f(x)|^{p} d x=\int_{\partial \mathbb{D}}|f(\psi(z))|^{p}\left|\psi^{\prime}(z)\right| d m(z) \\
& =\int_{\partial \mathbb{D}}\left|\left(\psi^{\prime}(z)\right)^{\gamma}(f \circ \psi)(z)\right|^{p} d m(z),
\end{aligned}
$$

where $d m\left(e^{i \theta}\right)=d \theta$ denotes arc-length measure on $\partial \mathbb{D}$. Thus $\left\|S_{\psi} f\right\|_{H^{p}(\mathbb{D})}=\|f\|_{H^{p}(\mathbb{U})}$.

Similarly, if $g \in L_{a}^{p}\left(\mathbb{D}, m_{\alpha}\right)$, then again by change of variables, we obtain

$$
\begin{aligned}
\|g\|_{L_{a}^{p}\left(m_{\alpha}\right)}^{p} & =\int_{\mathbb{D}}|g(z)|^{p}\left(1-|z|^{2}\right)^{\alpha} d A(z) \\
& =\int_{\mathbb{U}}\left|g\left(\psi^{-1}(\omega)\right)\right|\left(1-\left|\psi^{-1}(\omega)\right|^{2}\right)^{\alpha}\left|\left(\psi^{-1}\right)^{\prime}\right|^{2} d A(\omega),
\end{aligned}
$$


where $\left.\left(1-\left|\psi^{-1}(\omega)\right|^{2}\right)\right)=2\left|\left(\psi^{-1}\right)^{\prime}(\omega)\right| \mathfrak{I}(\omega)$. Thus

$$
\|g\|_{L_{a}^{p}\left(m_{\alpha}\right)}^{p}=2^{\alpha} \int_{\mathbb{U}}\left|\left(\psi^{-1}\right)^{\prime}\right|^{\alpha+2}\left|g \circ \psi^{-1}\right|^{p}(\mathfrak{I}(\omega))^{\alpha} d A(\omega)=2^{\alpha}\left\|S_{\psi^{-1}} g\right\|_{L_{a}^{p}\left(\mu_{\alpha}\right)}^{p} .
$$

If $g \in H^{p}(\mathbb{D})$, then

$$
\begin{aligned}
\left\|g^{p}\right\|_{H^{p}(\mathbb{D})} & =\int_{\partial \mathbb{D}}|g(z)|^{p} d m(z)=\int_{\mathbb{R}}\left|g\left(\psi^{-1}(x)\right)\right|^{p}\left|\left(\psi^{-1}\right)^{\prime}\right| d x \\
& =\left\|S_{\psi^{-1}} g\right\|_{H^{p}(\mathbb{R})}^{p} .
\end{aligned}
$$

Lemma 1.2. Let $X(\mathbb{D})$ denote either $H^{p}(\mathbb{D})$ or $L_{a}^{p}\left(\mathbb{D}, m_{\alpha}\right), 1 \leq p<\infty$ and $\alpha>-1$. Let $\gamma=\frac{\alpha+2}{p}(\alpha=-1$ in case $X(\mathbb{D})=$ $\left.H^{p}(\mathbb{D})\right)$. Then there exists a constant $C=C_{X(\mathbb{D})}$ such that for every $f \in X(\mathbb{D})$ and $z \in \mathbb{D}$,

$$
|f(z)| \leq \frac{C\|f\|_{X(\mathbb{D})}}{\left(1-|z|^{2}\right)^{\gamma}}
$$

Proof. We begin by showing that $|f(0)| \leq C\|f\|$. Let $f \in H^{p}(\mathbb{D})$. Then $\forall r, 0<r<1$, the mean value property implies that $f(0)=\frac{1}{2 \pi} \int_{0}^{2 \pi} f\left(r e^{i \theta}\right) d \theta$. Thus $|f(0)| \leq \frac{1}{2 \pi} \int_{0}^{2 \pi}\left|f\left(r e^{i \theta}\right)\right| d \theta$ and Jensen's inequality implies

$$
|f(0)|^{p} \leq \frac{1}{2 \pi} \int_{0}^{2 \pi}\left|f\left(r e^{i \theta}\right)\right|^{p} d \theta \leq\|f\|_{H^{p}(\mathbb{D})}^{p} .
$$

Similarly, if $f \in L_{a}^{p}\left(m_{\alpha}\right)$, then $\forall r, 0<r<1,|f(0)|^{p} \leq \frac{1}{2 \pi} \int_{0}^{2 \pi}\left|f\left(r e^{i \theta}\right)\right|^{p} d \theta$. Thus

$$
|f(0)|^{p} \int_{0}^{1}\left(1-r^{2}\right)^{\alpha} 2 r d r \leq \int_{0}^{1}\left(1-r^{2}\right)^{\alpha} 2 r d r \frac{1}{2 \pi} \int_{0}^{2 \pi}\left|f\left(r e^{i \theta}\right)\right|^{p} d \theta=\|f\|_{L_{a}^{p}\left(m_{\alpha}\right)} .
$$

If $a \in \mathbb{D}$, let $\phi_{a}(z)=\frac{a-z}{1-\bar{a} z} \in \operatorname{Aut}(\mathbb{D})$, where Aut $(\mathbb{D})$ denotes the group of automorphisms of $\mathbb{D}$. Then $S_{\phi_{a}} f:=\left(\phi_{a}^{\prime}\right)^{\gamma} f \circ \phi_{a}$ is an isometry on $X(\mathbb{D})$.

Indeed, in the Hardy space case,

$$
\left\|S_{\phi_{a}} f\right\|_{H^{p}(\mathbb{D})}^{p}=\int_{0}^{2 \pi}\left|f\left(\phi_{a}\left(e^{i \theta}\right)\right)\right|^{p}\left|\phi_{a}^{\prime}\left(e^{i \theta}\right) i e^{i \theta}\right| d \theta=\int_{0}^{2 \pi}\left|f\left(e^{i t}\right)\right|^{p} d t .
$$

In the Bergman space case, we note that, by the Schwarz-Pick Lemma [2, Lemma I.1.2], $\left(1-|z|^{2}\right)\left|\phi_{a}^{\prime}(z)\right|=1-\left|\phi_{a}(z)\right|^{2} \forall z \in \mathbb{D}$, and therefore a change of variables argument implies

$$
\begin{aligned}
\left\|S_{\phi_{a}} f\right\|_{L_{a}^{p}\left(m_{\alpha}\right)}^{p} & =\int_{\mathbb{D}}\left|f\left(\phi_{a}(z)\right)\right|^{p}\left(\left(1-|z|^{2}\right)\left|\phi_{a}^{\prime}\right|\right)^{\alpha}\left|\phi_{a}^{\prime}\right| d A(z) \\
& =\int_{\mathbb{D}}|f(\omega)|^{p}\left(1-|\omega|^{2}\right)^{\alpha} d A(\omega)=\|f\|_{L_{a}^{p}\left(m_{\alpha}\right)}^{p} .
\end{aligned}
$$

Thus if $a \in \mathbb{D},\left|\phi_{a}^{\prime}(0)\right|^{\gamma}|f(a)|=\left|S_{\phi_{a}} f(0)\right| \leq C\|f\|$ or $|f(a)| \leq \frac{C\|f\|}{(1-|a|)^{\gamma}}$, as claimed.

The following is an immediate consequence of the above Lemmas,

Corollary 1.3. Let $X$ denote either $H^{p}(\mathbb{U})$ or $L_{a}^{p}\left(\mu_{\alpha}\right), 1 \leq p<\infty$ and $\alpha>-1$. Let $\gamma=\frac{\alpha+2}{p}\left(\alpha=-1\right.$ in case $\left.X=H^{p}(\mathbb{U})\right)$. Then there exists a constant $C=C_{X}$ such that for every $f \in X$ and $z \in \mathbb{U}$,

$$
|f(z)| \leq \frac{C\|f\|_{X}}{(\mathfrak{I}(z))^{\gamma}}
$$

Proof. Let $g=S_{\psi} f$. Then by Proposition 1.1 above, $\|g\|_{H^{p}}=\|f\|_{H^{p}}$ and $\|g\|_{L_{a}^{p}\left(m_{\alpha}\right)}=2^{-\frac{\alpha}{p}}\|f\|_{L_{a}^{p}\left(\mu_{\alpha}\right)}$. Now, if $a=\psi^{-1}(z)$, then $\left(1-|a|^{2}\right)\left|\psi^{\prime}(a)\right|=2 \mathfrak{I}(z)$ and

$$
\left|\psi^{\prime}(a)\right|^{\gamma}|f(z)| \leq|g(a)| \leq \frac{C\|f\|}{\left(1-|a|^{2}\right)^{\gamma}},
$$

implying that $|f(z)| \leq \frac{C\|f\|}{(2 \mathfrak{S}(z))^{\gamma}}$. 
As consequence of the growth conditions given by equations (1.1) and (1.2), both Hardy and Bergman spaces $(X$ and $X(\mathbb{D}))$ are Banach spaces. In fact, if $p=2$, it turns out that these spaces are Hilbert spaces, and moreover, the Bergman space $L_{a}^{p}(\cdot)$ is a closed subspace of the classical Lebesgue space $L^{p}(\cdot)$. For a detailed theory of Hardy spaces, we refer to [2, 3, 4], while for Bergman spaces, see $[4,5,6,7,8]$.

The reproducing kernels for Hardy and Bergman spaces of the unit disk $\mathbb{D}$ are well known in literature. See for instance [3] for Hardy spaces, and $[4,8]$ for Bergman spaces. The corresponding reproducing kernels for the Hardy and Bergman spaces of the upper half plane $\mathbb{U}$ is not well captured in literature. In general, the theory of analytic spaces of the upper half plane is much less complete compared to the unit disk setting. In this paper, we determine explicitly the reproducing kernels on $\mathbb{U}$ for the two spaces. As a result, we also give the corresponding projections and consequently, establish some known duality properties of these spaces. These results are part of my Ph.D. dissertation [9].

\section{Reproducing Kernels on the upper half plane}

Let $\mathscr{H}$ denote a Hilbert space of functions defined on an open set $\Omega \subset \mathbb{C}$. We call a reproducing kernel for $\mathscr{H}$, a complex function $K: \Omega \times \Omega \rightarrow \mathbb{C}$ such that, if we put $K_{\omega}(z):=K(z, \omega)$, then the following two properties hold:

1. for every $\omega \in \Omega$, the function $K_{\omega}$ belongs to $\mathscr{H}$, and

2. for all $f \in \mathscr{H}$ and $\omega \in \Omega$, we have

$$
f(\omega)=\left\langle f, K_{\omega}\right\rangle .
$$

It is clear that the above two properties imply that such a kernel $K$ satisfies the identity $K(z, \omega)=\overline{K(\omega, z)}$ for all $z, \omega \in \Omega$. Indeed

$$
\begin{aligned}
K(z, \omega)=K_{\omega}(z) & =\left\langle K_{\omega}, K_{z}\right\rangle \\
& =\overline{\left\langle K_{z}, K_{\omega}\right\rangle}=\overline{K_{z}(\omega)}=\overline{K(\omega, z)} .
\end{aligned}
$$

The growth condition estimates ( for $X$ and $X(\mathbb{D})$ ) given by equations (1.1) and (1.2) and the Riesz representation theorem for $\mathscr{H}^{*}$, imply that $H^{2}(\cdot)$ and $L_{a}^{2}(\cdot)$ are reproducing kernel Hilbert spaces.

\subsection{Bergman spaces of the upper half plane}

For the Bergman spaces (weighted), the reproducing kernel is also called the Bergman kernel (weighted). In particular, for the weighted Bergman spaces, we denote the weighted Bergman kernels by $K_{\alpha, \mathbb{D}}$ and $K_{\alpha, \mathbb{U}}$ on $\mathbb{D}$ and $\mathbb{U}$, respectively. If the setting $\mathbb{D}$ or $\mathbb{U}$ is understood, we simply write $K_{\alpha}$.

For Bergman spaces of the unit disc, $L_{a}^{p}\left(\mathbb{D}, m_{\alpha}\right), \alpha>-1$, the weighted Bergman kernel has been computed in [8] and is given by

$$
K_{\alpha, \mathbb{D}}(z, \omega)=\frac{1}{(1-z \bar{\omega})^{2+\alpha}} .
$$

The function $K_{\alpha}$ on the unit disc $\mathbb{D}$ has been exhaustively studied in literature and some of its properties, for example, boundedness have far reaching consequences in the theory of analytic functions in $L_{a}^{p}\left(\mathbb{D}, m_{\alpha}\right)$. For a comprehensive theory of Bergman kernels and hence projections on $\mathbb{D}$, see for instance [4] or [8], and references therein.

In this section, we shall present the corresponding theory of the Bergman kernels and projections on the upper half-plane $\mathbb{U}$. In the next result, we compute explicitly the weighted Bergman kernel on the upper half-plane $\mathbb{U}$, which we denote by $K_{\alpha}=K_{\alpha, \mathbb{U}}$, and is acting on the Hilbert space $L_{a}^{2}\left(\mathbb{U}, \mu_{\alpha}\right)$.

Theorem 2.1. If $\alpha>-1$, then the weighted Bergman kernel of $L_{a}^{2}\left(\mathbb{U}, \mu_{\alpha}\right)$ is given by

$$
K_{\alpha}(z, \omega)=\frac{2^{\alpha}}{[-i(z-\bar{\omega})]^{2+\alpha}} .
$$

Proof. Let $K_{\alpha, \mathbb{U}}, K_{\alpha, \mathbb{D}}$ be the weighted Bergman kernels of $L_{a}^{2}\left(\mathbb{U}, \mu_{\alpha}\right)$ and $L_{a}^{2}\left(\mathbb{D}, m_{\alpha}\right)$ respectively. Then $K_{\alpha, \mathbb{D}}$ is given by equation (2.1). We need to compute $K_{\alpha, \mathbb{U}}$. The Cayley transform $\psi(z)=\frac{i(1+z)}{1-z}$ maps $\mathbb{U}$ conformally onto $\mathbb{D}$ with inverse, $\psi^{-1}(\omega)=\frac{\omega-i}{\omega+i}$. It follows from Proposition 1.1 that $T f(\xi):=2^{-\frac{\alpha}{2}}\left(\psi^{\prime}(\xi)\right)^{1+\frac{\alpha}{2}} f(\psi(\xi))$ is an isometric surjective isomorphism 
of $L_{a}^{2}\left(\mathbb{U}, \mu_{\alpha}\right)$ onto $L_{a}^{2}\left(\mathbb{D}, m_{\alpha}\right)$. Since $L_{a}^{2}(\cdot)$ is a Hilbert space, it then follows that $T$ is unitary, that is, $T^{*}=T^{-1}$. For every $\xi \in \mathbb{D}$, and by writing $K_{\alpha, \mathbb{D}, \xi}=K_{\alpha, \mathbb{D}}(\cdot, \xi)$ simply as $K_{\mathbb{D}, \xi}$, we have using the definition of $K_{\alpha}$,

$$
\begin{aligned}
T f(\xi) & =2^{-\frac{\alpha}{2}}\left(\psi^{\prime}(\xi)\right)^{1+\frac{\alpha}{2}} f(\psi(\xi))=2^{-\frac{\alpha}{2}} \frac{(2 i)^{1+\frac{\alpha}{2}}}{(1-\xi)^{2+\alpha}} f(\psi(\xi)), \\
& =\left\langle T f, K_{\mathbb{D}, \xi}\right\rangle_{L_{a}^{2}\left(\mathbb{D}, m_{\alpha}\right)}=\left\langle f, T^{-1} K_{\mathbb{D}, \xi}\right\rangle_{L_{a}^{2}\left(\mathbb{U}, \mu_{\alpha}\right)} .
\end{aligned}
$$

We now compute $T^{-1} K_{\mathbb{D}, \xi}$. For $z \in \mathbb{U}$, we have

$$
\left(T^{-1} K_{\mathbb{D}, \xi}\right)(z)=2^{\frac{\alpha}{2}}\left(\left(\psi^{-1}(z)\right)^{\prime}\right)^{1+\frac{\alpha}{2}} K_{\mathbb{D}, \xi}\left(\psi^{-1}(z)\right) .
$$

But by equation (2.1),

$$
K_{\mathbb{D}, \xi}\left(\psi^{-1}(z)\right)=\frac{1}{\left(1-\frac{z-i}{z+i} \bar{\xi}\right)^{2+\alpha}}=\frac{(z+i)^{2+\alpha}}{(1-\bar{\xi})^{2+\alpha}[z-\overline{\psi(\xi)}]} .
$$

Therefore,

$$
\begin{aligned}
2^{-\frac{\alpha}{2}}\left(\psi^{\prime}(\xi)\right)^{1+\frac{\alpha}{2}} f(\psi(\xi)) & =T f(\xi)=\left\langle f, T^{-1} K_{\mathbb{D}, \xi}\right\rangle_{L_{a}^{2}\left(\mathbb{U}, \mu_{\alpha}\right)} \\
& =2^{\frac{\alpha}{2}} \frac{(-2 i)^{1+\frac{\alpha}{2}}}{(1-\bar{\xi})^{2+\alpha}}\left\langle f, \frac{1}{(z-\overline{\psi(\xi)})^{2+\alpha}}\right\rangle,
\end{aligned}
$$

which implies that $f(\psi(\xi))=2^{\alpha}\left\langle f, \frac{1}{[-i(z-\overline{\psi(\xi)})]^{2+\alpha}}\right\rangle$, and thus,

$$
f(\omega)=\left\langle f, \frac{2^{\alpha}}{[-i(z-\bar{\omega})]^{2+\alpha}}\right\rangle, \quad \omega \in \mathbb{U} .
$$

In particular, if we write $\psi(\xi)=\omega$, then

$$
K_{\mathbb{U}, \omega}(z)=\frac{2^{\alpha}}{[-i(z-\bar{\omega})]^{2+\alpha}}, \quad \text { as desired. }
$$

It is important to note that a similar formula has been obtained through the use of Paley-Weiener theorem which in itself involves Fourier transform, see [5]. We consider the method applied in this paper to be more direct and simple. Since $L_{a}^{2}\left(\mathbb{U}, \mu_{\alpha}\right)$ is a closed subspace of the Hilbert space $L^{2}\left(\mathbb{U}, \mu_{\alpha}\right)$, there exists an orthogonal projection $P_{\alpha}: L^{2}\left(\mathbb{U}, \mu_{\alpha}\right) \rightarrow L_{a}^{2}\left(\mathbb{U}, \mu_{\alpha}\right)$ which we shall call the weighted Bergman projection on $L^{2}\left(\mathbb{U}, \mu_{\alpha}\right)$.

Proposition 2.2. The weighted Bergman projection $P_{\alpha}$ from $L^{2}\left(\mathbb{U}, \mu_{\alpha}\right)$ onto the subspace $L_{a}^{2}\left(\mathbb{U}, \mu_{\alpha}\right)$ is given explicitly by

$$
P_{\alpha} f(z)=\int_{\mathbb{U}} K_{\alpha}(z, \omega) f(\omega) d \mu_{\alpha}(\omega),
$$

where $K_{\alpha}$ is the weighted Bergman kernel on the half plane given by equation (2.2).

Proof. Indeed, by the reproducing property of $K_{\alpha}(z, \omega)$ and the self - adjointness of $P_{\alpha}$ on $L^{2}\left(\mathbb{U}, \mu_{\alpha}\right)$, we have

$$
\begin{aligned}
P_{\alpha} f(z) & =\left\langle P_{\alpha} f, K_{\alpha}(., z)\right\rangle_{L_{a}^{2}\left(\mu_{\alpha}\right)}=\left\langle f, P_{\alpha} K_{\alpha}(., z)\right\rangle_{L_{a}^{2}\left(\mu_{\alpha}\right)} \\
& =\left\langle f, K_{\alpha}(., z)\right\rangle_{L_{a}^{2}\left(\mu_{\alpha}\right)}=\int_{\mathbb{U}} K_{\alpha}(z, \omega) f(\omega) d \mu_{\alpha}(\omega), \quad \text { as claimed. }
\end{aligned}
$$

At this point, it is natural to ask whether the Bergman projection $P_{\alpha}$ extends in some meaningful way to $L_{a}^{p}\left(\mathbb{U}, \mu_{\alpha}\right)$ for the case $p \neq 2$, and in that case, whether the reproducing property of $K_{\alpha}(z, \omega)$, (that is, $P_{\alpha} F=F$ ) holds in $L_{a}^{p}\left(\mathbb{U}, \mu_{\alpha}\right)$. These questions were posed in [5]. In this section, we address these questions but first we prove some elementary results that will be useful in the sequel. 
Proposition 2.3. Let $X=H^{p}(\mathbb{U})$ or $L_{a}^{p}\left(\mathbb{U}, \mu_{\alpha}\right), 1 \leq p<\infty$. Let $a>0, b \in \mathbb{R}$ and define $T f(z)=f(a z+b)$ for every $f \in X$, then $\|T\| \leq a^{-\gamma}$.

Proof. If $f \in L_{a}^{p}\left(\mathbb{U}, \mu_{\alpha}\right)$, then

$$
\begin{aligned}
\|T f\|_{p}^{p} & =\int_{\mathbb{U}}|f(a z+b)|^{p} \frac{(\mathfrak{I}(a z+b))^{\alpha}}{a^{\alpha+2}}|a|^{2} d A(z) \\
& =\int_{a \mathbb{U}}|f(\omega)|^{p}(\mathfrak{I}(\omega))^{\alpha} d A(\omega) a^{-(\alpha+2)},
\end{aligned}
$$

and if $f \in H^{p}(\mathbb{U})$, then

$$
\begin{aligned}
\|T f\|_{p}^{p} & =\sup _{y>0} \int_{-\infty}^{\infty}|f(a x+i a y+b)|^{p} d x=\sup _{t>0} \int_{-\infty}^{\infty}|f(a x+b+i t)|^{p} d x \\
& =\frac{1}{a} \sup _{t>0} \int_{-\infty}^{\infty}|f(s+i t)|^{p} d s=\frac{1}{a}\|f\|_{p}^{p} .
\end{aligned}
$$

The next two Lemmas give examples of analytic functions and the conditions they must satisfy to belong to the spaces $X$ and $X(\mathbb{D})$.

Lemma 2.4. Let $X(\mathbb{D})$ denote one of the spaces $H^{p}(\mathbb{D})$ or $L_{a}^{p}\left(\mathbb{D}, m_{\alpha}\right), 1 \leq p<\infty$ and $\alpha>-1\left(\alpha=-1\right.$ if $\left.X(\mathbb{D})=H^{p}(\mathbb{D})\right)$, and let $\gamma=(\alpha+2) / p$. Then for $\eta \in \mathbb{C}$,

$$
\left(e^{i \theta}-z\right)^{\eta} \in X(\mathbb{D}) \text { if and only if } \Re \eta>-\gamma .
$$

Proof. We first consider the Bergman space case, that is $X(\mathbb{D})=L_{a}^{p}\left(\mathbb{D}, m_{\alpha}\right)$.

Recall $\left(e^{i \theta}-z\right)^{\eta} \in L_{a}^{p}\left(\mathbb{D}, m_{\alpha}\right) \Leftrightarrow \int_{\mathbb{D}}\left|\left(e^{i \theta}-z\right)^{\eta}\right|^{p} d m_{\alpha}(z)<\infty$. Now,

$$
\begin{aligned}
\int_{\mathbb{D}}\left|\left(e^{i \theta}-z\right)^{\eta}\right|^{p} d m_{\alpha}(z) & =\int_{\mathbb{D}}\left|\left(e^{i \theta}-z\right)^{\eta}\right|^{p}\left(1-|z|^{2}\right)^{\alpha} d A(z) \\
& =\int_{\mathbb{D}}\left|1-z e^{-i \theta}\right|^{p \Re(\eta)}\left(1-|z|^{2}\right)^{\alpha} d A(z) \\
& =\int_{\mathbb{D}} \frac{\left(1-|z|^{2}\right)^{\alpha}}{\left|1-z e^{-i \theta}\right|^{-p \Re(\eta)}} d A(z) .
\end{aligned}
$$

It then follows immediately from [8, Lemma 3.10] that equation (2.4) is bounded if and only if $-p \Re(\eta)-\alpha-2<0$, that is, $\Re(\eta)>-\frac{\alpha+2}{p}$, as desired.

For $X(\mathbb{D})=H^{p}(\mathbb{D})$, we use the fact that functions in $H^{p}(\mathbb{D})$ can be identified with their boundary values. Fix $\theta \in \mathbb{R}$ and let $f(z)=\left(e^{i \theta}-z\right)^{\eta}$. Then $f$ has boundary values $f\left(e^{i t}\right)=\left(e^{i \theta}-e^{i t}\right)^{\eta}$ and $f \in H^{p}(\mathbb{D})$ is equivalent to

$$
\begin{aligned}
\int_{-\pi}^{\pi}\left|f\left(e^{i t}\right)\right|^{p} d t & =\int_{\theta-\pi}^{\theta+\pi}\left|\left(1-e^{i(t-\theta)}\right)^{\eta}\right|^{p} d t \\
& =\int_{-\pi}^{\pi} 2^{p \Re(\eta)}|\sin (t / 2)|^{p \Re(\eta)} d t<\infty .
\end{aligned}
$$

But the equation (2.5) holds if and only if $p \Re(\eta)>-1$, as claimed.

Lemma 2.5. Let $X$ denote one of the spaces $H^{p}(\mathbb{U})$ or $L_{a}^{p}\left(\mathbb{U}, \mu_{\alpha}\right), 1 \leq p<\infty$ and $\alpha>-1\left(\alpha=-1\right.$ if $\left.X=H^{p}(\mathbb{U})\right)$, and let $\gamma=(\alpha+2) / p$. If $c \in \mathbb{R}$ and $\lambda, v \in \mathbb{C}$, then

1. $f(\omega)=(\omega-c)^{\lambda}(\omega+i)^{v} \in X$ if and only if $\Re(\lambda+v)<-\gamma<\Re(\lambda)$. In particular, $(\omega-c)^{\lambda} \notin X$ for any $\lambda \in \mathbb{C}$, and $(\omega+i)^{v} \in X$ if and only if $\Re v<-\gamma$.

2. $f(\omega)=e^{i \omega} / \omega^{c} \in X$ if and only if $1 / p<c<\gamma$. In particular, $e^{i \omega} / \omega^{c} \notin H^{p}(\mathbb{U})$ for any $c \in \mathbb{R}$.

Proof. See [10, Lemma 3.2].

We now give the following proposition, 
Proposition 2.6. Let $X=H^{p}(\mathbb{U})$ or $L_{a}^{p}\left(\mathbb{U}, \mu_{\alpha}\right)$. If $v>0$, then

$(\omega+i v)^{v} \in X$ if and only if $v>-\gamma$.

Proof. If $f(\omega)=(\omega+i v)^{v}$, then

$$
f(\omega)=v^{v}\left(\frac{1}{v} \omega+i\right)^{v}=v^{v} T g(\omega),
$$

where $T h(z)=h\left(\frac{1}{v} z\right)$ and $g(\omega)=(\omega+i)^{v}$. Now Proposition 2.3 and Lemma 2.5 immediately yield the desired result.

To begin addressing the questions mentioned earlier in this section concerning the extension of the Bergman kernel $K_{\alpha}$ to the cases $p \neq 2$, we give the following direct consequence of the above proposition.

Corollary 2.7. For fixed $\omega \in \mathbb{U}$, the Bergman projection $P_{\alpha}$ belongs to $L_{a}^{q}\left(\mathbb{U}, \mu_{\alpha}\right)$ if and only if $1<q \leq \infty$.

Proof. If $K_{\alpha}(z, \omega)=\frac{2^{\alpha}}{(-i(z-\bar{\omega}))^{\alpha+2}},(z, \omega \in \mathbb{U})$, then for fixed $\omega \in \mathbb{U}$,

$$
K_{\alpha}(z, \omega)=2^{\alpha} i^{\alpha+2}((z-\Re(\omega))+i \mathfrak{I}(\omega))^{-(\alpha+2)} .
$$

Therefore by Proposition 2.6, $K_{\alpha}(\cdot, \omega) \in L_{a}^{q}\left(\mathbb{U}, \mu_{\alpha}\right)$ if and only if $-(\alpha+2)<-(\alpha+2) / q$, which is equivalent to $q>1$. Moreover, if $z=x+i y, y>0$, we have

$$
\left|K_{\alpha}(z, \omega)\right| \leq \frac{2^{\alpha}}{(\mathfrak{I}(\omega))^{\alpha+2}}
$$

implying that $K_{\alpha}(\cdot, \omega) \in L_{a}^{\infty}\left(\mathbb{U}, \mu_{\alpha}\right)$.

We can now prove the following result;

Proposition 2.8. Let $1 \leq p<\infty$, then for each $f \in L_{a}^{p}\left(\mathbb{U}, \mu_{\alpha}\right)$,

$$
f(z)=\int_{\mathbb{U}} f(\omega) K_{\alpha}(z, \omega) d \mu_{\alpha}(\omega) .
$$

Proof. If $f \in L_{a}^{p}\left(\mathbb{U}, \mu_{\alpha}\right), 1 \leq p<\infty$, then by Corollary $2.7, K_{\alpha}(\cdot, z) \in L_{a}^{p}\left(\mu_{\alpha}\right), \frac{1}{p}+\frac{1}{q}=1$ and so Hölder's inequality implies that

$$
f \longmapsto \int_{\mathbb{U}} f(\omega) K_{\alpha}(z, \omega) d \mu_{\alpha}(\omega)=\left\langle f, K_{\alpha}(\omega, z)\right\rangle
$$

is continuous; moreover, by the reproducing property,

$$
f(z)=\int_{\mathbb{U}} f(\omega) K_{\alpha}(z, \omega) d \mu_{\alpha}(\omega) \text { for all } f \in L_{a}^{p}\left(\mu_{\alpha}\right) \cap L_{a}^{2}\left(\mu_{\alpha}\right) .
$$

Since $L_{a}^{p}\left(\mu_{\alpha}\right) \cap L_{a}^{2}\left(\mu_{\alpha}\right)$ is dense in $L_{a}^{p}\left(\mu_{\alpha}\right)$, we're done.

The following theorem characterizes when $P_{\alpha}$ is a bounded projection from $L^{p}\left(\mathbb{U}, \mu_{\alpha}\right)$ onto $L_{a}^{p}\left(\mathbb{U}, \mu_{\alpha}\right)$, see D. Békollé, et.al. [5] for the details.

Theorem 2.9. The Bergman projection

$$
P_{\alpha} f(z):=\int_{\mathbb{U}} f(\omega) K_{\alpha}(z, \omega) d \mu_{\alpha}(\omega), \quad \alpha>-1,
$$

is a bounded projection from $L^{p}\left(\mathbb{U}, \mu_{\alpha}\right)$ onto $L_{a}^{p}\left(\mathbb{U}, \mu_{\alpha}\right)$ if and only if $1<p<\infty$.

An immediate consequence of the boundedness of the Bergman projection $P_{\alpha}$ on $L_{a}^{p}\left(\mathbb{U}, \mu_{\alpha}\right)$ is the duality of Bergman spaces $L_{a}^{p}\left(\mathbb{U}, \mu_{\alpha}\right)$ which we give in the following result, 
Corollary 2.10. Let $1<p<\infty$ and $q$ be conjugate to $p$ in the sense that $\frac{1}{p}+\frac{1}{q}=1$. Let $\left(L_{a}^{p}\left(\mathbb{U}, \mu_{\alpha}\right)\right)^{*}$ be the dual space of $L_{a}^{p}\left(\mathbb{U}, \mu_{\alpha}\right)$, then

$$
\left(L_{a}^{p}\left(\mathbb{U}, \mu_{\alpha}\right)\right)^{*} \approx L_{a}^{q}\left(\mathbb{U}, \mu_{\alpha}\right), \quad \alpha>-1,
$$

under the sesquilinear pairing

$$
\langle f, g\rangle=\int_{\mathbb{U}} f(\omega) \overline{g(\omega)} d \mu_{\alpha} \quad\left(f \in L_{a}^{p}\left(\mu_{\alpha}\right), g \in L_{a}^{q}\left(\mu_{\alpha}\right)\right) .
$$

Proof. The classical duality between $L^{p}$ - spaces gives

$$
\left(L^{p}\left(\mathbb{U}, \mu_{\alpha}\right)\right)^{*} \approx L^{q}\left(\mathbb{U}, \mu_{\alpha}\right) .
$$

By Hahn-Banach extension theorem and the boundedness of the Bergman projection $P_{\alpha}$ for $1<p<\infty$, (Theorem 2.9), we have

$$
\left(L_{a}^{p}\left(\mathbb{U}, \mu_{\alpha}\right)\right)^{*}=P_{\alpha}\left(L^{p}\left(\mathbb{U}, \mu_{\alpha}\right)\right)^{*} \approx P_{\alpha} L^{q}\left(\mathbb{U}, \mu_{\alpha}\right)=L_{a}^{q}\left(\mathbb{U}, \mu_{\alpha}\right), \quad \text { as desired. }
$$

It is important to take note that under the above duality pairing, see equation (2.7), the adjoint operator is conjugate linear. Moreover, $L_{a}^{p}\left(\mu_{\alpha}\right)$ spaces for $1<p<\infty$ are reflexive and thus;

$$
\left(L_{a}^{q}\left(\mu_{\alpha}\right)\right)^{*} \approx\left(L_{a}^{p}\left(\mu_{\alpha}\right)\right)^{* *} \approx L_{a}^{p}\left(\mu_{\alpha}\right) .
$$

\subsection{Hardy spaces of the upper half plane}

The reproducing kernel for Hardy spaces is also called the Cauchy - Szegö kernel or simply the Szegö kernel, with the corresponding projection called the Cauchy - Szegö projection or simply the Szegö projection. We refer to [6] or [11, Chapter 8] for a good account of the theory of the Szegö kernel and projection on Hardy spaces. Recall that functions in $H^{p}(\mathbb{D})$ have boundary values almost everywhere in $L_{a}^{p}(\partial \mathbb{D})$ and that if $1 \leq p<\infty$, then $H^{p}(\mathbb{D})=\mathrm{cl}_{L^{p}}(\partial \mathbb{D}) \mathbb{C}[z]$, where $\mathbb{C}[z]$ denotes analytic polynomials in $z$, and $\mathrm{cl}_{L^{p}(\partial \mathbb{D})}$ is the $L^{p}(\partial \mathbb{D})$-closure. In fact the Hilbert space $H^{2}(\mathbb{D})$ has orthonormal basis $\left(z^{n}\right)_{n \geq 0}$.

As noted in [6, Chapter 2], Cauchy's theorem implies that the reproducing kernel for $H^{1}(\mathbb{D})$ is given by

$$
S_{\mathbb{D}}(z, \omega)=\frac{1}{1-z \bar{\omega}} \quad(z \in \partial \mathbb{D}, \omega \in \mathbb{D}) .
$$

Therefore, the Cauchy-Szegö projection $P_{\mathbb{D}}$ is given by

$$
\begin{aligned}
P_{\mathbb{D}} \varphi(z) & =\langle\varphi, S(\omega, z)\rangle=\int_{\partial \mathbb{D}} \varphi(\omega) \overline{S_{\mathbb{D}}(\omega, z)} d m(\omega) \\
& =\frac{1}{2 \pi} \int_{0}^{2 \pi} \frac{\varphi\left(e^{i t}\right)}{1-e^{-i t} z} d t
\end{aligned}
$$

and satisfies $P_{\mathbb{D}} f=f$ for all $f \in H^{1}(\mathbb{D})$. The following theorem whose details can be found in [6, Chapter 2] characterizes the boundedness of the Szegö projection for the case when $p \neq 1$.

Theorem 2.11. If $1<p<\infty$, then $P_{\mathbb{D}}: L^{p}(\partial \mathbb{D}) \rightarrow H^{p}(\mathbb{D})$ is bounded and surjective.

In the next theorem, we establish the corresponding Cauchy-Szegö kernel on the upper half plane.

Theorem 2.12. The Cauchy - Szegö kernel for $H^{2}(\mathbb{U})$ is given by

$$
S_{\mathbb{U}}(z, \xi)=\frac{i}{z-\bar{\xi}} .
$$

Proof. Let $T: H^{p}(\mathbb{U}) \rightarrow H^{p}(\mathbb{D})$ be given by $T f(z)=\left(\psi^{\prime}(z)\right)^{1 / p} f(\psi(z))$. Then by Proposition 1.1, $T$ is surjective isometry. In particular, $T: H^{2}(\mathbb{U}) \rightarrow H^{2}(\mathbb{D})$ is unitary with $T^{*}=T^{-1}$, and $T^{-1} g(\omega)=\left(\left(\psi^{-1}\right)^{\prime}(\omega)\right)^{1 / 2} g\left(\psi^{-1}(\omega)\right)$, where $\psi$ is the Cayley transform. We wish to compute the corresponding Szegö kernel on the upper half-plane, $\mathbb{U}$ : Let $\xi \in \mathbb{U}, z=\psi^{-1}(\xi) \in \mathbb{D}$. Also, let $f \in H^{2}(\mathbb{U})$ and $g=T f$. Then

$$
\begin{aligned}
g(z) & =\int_{\partial \mathbb{D}} g(\omega) S_{\mathbb{D}}(z, \omega) d m(\omega)=\langle g, S(\cdot, z)\rangle_{\partial \mathbb{D}}=\left\langle T f, S_{\mathbb{D}, z}\right\rangle_{\partial \mathbb{D}} \\
& =\left\langle f, T^{*} S_{\mathbb{D}, z}\right\rangle_{\mathbb{R}} .
\end{aligned}
$$


But we have

$$
g(z)=g\left(\psi^{-1}(\xi)\right)=T f\left(\psi^{-1}(\xi)\right)=\left(\psi^{\prime}\left(\psi^{-1}(\xi)\right)\right)^{1 / 2} f(\xi)=\frac{\xi+i}{(2 i)^{1 / 2}} f(\xi),
$$

and

$$
\begin{aligned}
\left(T^{*} S_{\mathbb{D}, z}\right)(\omega) & =\left(\left(\psi^{-1}\right)^{\prime}(\omega)\right)^{1 / 2} S_{\mathbb{D}}\left(\psi^{-1}(\omega), \psi^{-1}(\xi)\right) \\
& =\frac{(2 i)^{1 / 2}}{(\omega+i)}\left(\frac{1}{1-\left(\frac{\omega-1}{\omega+i}\right)\left(\frac{\bar{\xi}+i}{\bar{\xi}-i}\right)}\right) \\
& =\frac{(2 i)^{1 / 2}(\bar{\xi}-i)}{-2 i(\omega-\bar{\xi})} .
\end{aligned}
$$

Thus

$$
\begin{aligned}
f(\xi) & =\frac{(2 i)^{1 / 2}}{(\xi+i)} \int_{\mathbb{R}} f(x) \overline{\left(\frac{(2 i)^{1 / 2}(\bar{\xi}-i)}{2(-i)(x-\bar{\xi})}\right)} d x \\
& =\int_{\mathbb{R}} f(x) \overline{\left(\frac{(2 i)^{1 / 2}(-2 i)^{1 / 2}}{2(-i)(x-\bar{\xi})}\right)} d x .
\end{aligned}
$$

Therefore, for every $f \in H^{2}(\mathbb{U}), \xi \in \mathbb{U}$,

$$
f(\xi)=\int_{\mathbb{R}} f(x) \overline{\left(\frac{i}{x-\bar{\xi}}\right)} d x .
$$

Thus the Szegö kernel for $H^{2}(\mathbb{U})$ is given by

$$
S_{\mathbb{U}}(z, \xi)=\frac{i}{z-\bar{\xi}}
$$

Corollary 2.13. The Cauchy - Szegö projection P from $L^{2}(\mathbb{R})$ onto $H^{2}(\mathbb{U})$ is given explicitly by

$$
P \varphi(\xi)=\int_{\mathbb{R}} \varphi(x) S_{\mathbb{U}}(\xi, x) d x,
$$

where $S_{\mathbb{U}}$ is the Cauchy - Szegö kernel given by equation (2.9).

Proof. Adopting the above notation, we have

$$
\begin{aligned}
P \varphi(\xi) & =\int_{\mathbb{R}} \varphi(x) \overline{S_{\mathbb{U}}(x, \xi)} d x \\
& =\int_{\mathbb{R}} \varphi(x)\left(\frac{-i}{x-\xi}\right) d x \\
& =\int_{\mathbb{R}} \varphi(x) \frac{i}{\xi-x} d x \\
& =\int_{\mathbb{R}} \varphi(x) S_{\mathbb{U}}(\xi, x) d x .
\end{aligned}
$$

Therefore, the upper half-plane analogue of Theorem 2.11 is the following, 
Theorem 2.14. If $f \in H^{p}(\mathbb{U}), 1 \leq p<\infty$, then for every $\xi \in \mathbb{U}$,

$$
f(\xi)=\int_{\mathbb{R}} f(x) S_{\mathbb{U}}(\xi, x) d x,
$$

and if $p>1$, then the Szegö projection $P: L^{p}(\mathbb{R}) \rightarrow H^{p}(\mathbb{U})$ given by

$$
P \varphi(\xi)=\int_{\mathbb{R}} \varphi(x) S_{\mathbb{U}}(\xi, x) d x
$$

is bounded and surjective.

The boundedness of the Szegö projection $P$ on $L^{p}(\mathbb{R})$ given by Theorem 2.14 immediately yields the following duality of Hardy spaces $H^{p}(\mathbb{U})$, for $1<p<\infty$.

Corollary 2.15. Let $1<p<\infty$ and $q$ be such that $\frac{1}{p}+\frac{1}{q}=1$. Let $\left(H^{p}(\mathbb{U})\right)^{*}$ be the dual space of $H^{p}(\mathbb{U})$. Then

$$
\left(H^{p}(\mathbb{U})\right)^{*} \approx H^{q}(\mathbb{U}),
$$

via the sequilinear pairing

$$
\langle f, g\rangle=\int_{\mathbb{R}} f(x) \overline{g(x)} d x \quad\left(f \in H^{p}(\mathbb{U}), g \in H^{q}(\mathbb{U})\right) .
$$

Proof. It is well known that

$$
\left(L^{p}(\mathbb{R})\right)^{*} \approx L^{q}(\mathbb{R}) .
$$

Now, the Hahn-Banach extension theorem together with the boundedness of the Szegö projection $P$ for $1<p<\infty$ will yield,

$$
\left(H^{p}(\mathbb{U})\right)^{*} \approx\left(H^{p}(\mathbb{R})\right)^{*}=P\left(L^{p}(\mathbb{R})\right)^{*} \approx P L^{q}(\mathbb{R})=H^{q}(\mathbb{R}) \approx H^{q}(\mathbb{U}) .
$$

Again, we take note that under the pairing in equation (2.11), the adjoint operator from $\mathscr{L}(X)$ to $\mathscr{L}\left(X^{*}\right)$ is also conjugate linear. Since the Hardy spaces $H^{p}(\mathbb{U}), 1<p<\infty$, are reflexive Banach spaces, it follows that

$$
\left(H^{q}(\mathbb{U})\right)^{*} \approx\left(H^{p}(\mathbb{U})\right)^{* *} \approx H^{p}(\mathbb{U}) .
$$

\section{Acknowledgement}

I would like to thank the US department state through the Foreign Fulbright program for sponsoring my doctoral studies at Mississippi State University, United States. I would also wish to thank my home institution, Maseno University, Kenya, for the generous study leave.

\section{References}

[1] E. Albrecht, T. L. Miller, M. M. Neumann, Spectral properties of generalized Cesàro operators on Hardy and weighted Bergman spaces. Arch. Math. (Basel), 85 (2005), 446-459.

[2] J. B. Garnett, Bounded Analytic Functions. Graduate Texts in Mathematics, Revised First Edition, Springer, Berlin, 2010.

[3] P. Duren, Theory of $H^{p}$ spaces. Academic Press, New York, 1970.

[4] M. M. Peloso, Classical spaces of Holomorphic functions. Technical report, Universit di Milano, 2014.

[5] D. Békollé, A.Bonimi. G. Garrigós, C. Nana, M. Peloso, F. Ricci, Lecture notes on Bergman projections in tube domais over cones: an analytic and geometric viewpoint, IMHOTEP J. Afr. Math. Pures Appl. 5 (2004). http://webs.um.es/gustavo.garrigos/papers/workshop5.pdf

[6] P. Duren, A. Schuster, Bergman spaces. Mathematical Surveys and Monographs 100, Amer. Math. Soc., Providence, RI, 2004.

${ }^{\text {[7] }}$ H. Hedenmalm, B. Korenblum, K. Zhu, Theory of Bergman spaces. Springer Verlag, New York, Inc., 2000. 
${ }^{[8]}$ K. Zhu, Operator theory in function spaces. Mathematical Surveys and Monographs 138, Amer. Math. Soc., Providence, 2007.

[9] J. O. Bonyo, Groups of isometries associated with automorphisms of the half plane. Ph.D. dissertation, Mississippi State University, USA, 2015.

[10] S. Ballamoole, J. O. Bonyo, T. L. Miller, V. G. Miller, Cesàro operators on the Hardy and Bergman spaces of the half plane. Complex Anal. Oper. Theory 10 (2016), 187-203.

[11] K. Hoffman, Banach spaces of analytic functions. Prentice - Hall, Inc., Englewood Cliffs, N.J., 1962. 